\title{
Voluntary and Involuntary Nonadherence: Terminology for Labeling Patient Participation
}

\author{
RYAN PFERDEHIRT, D.BIOETHICS \\ rpferdehirt@practicalbioethics.org
}

\begin{abstract}
The descriptions and labels healthcare providers place upon patients can greatly determine the quality and availability of the healthcare they receive. When that patient is given the label nonadherent or noncompliant within healthcare, it can have a detrimental impact on the patient. But patient adherence is a more complicated issue than can be express with a binary determination; is or is not, yes or no. Therefore, additional terminology should be utilized to properly convey the patient's participation status when the patient does not fully engage in care. This paper proposes the implementation of additional qualifying language: involuntary nonadherence and voluntary nonadherence. Involuntary nonadherence is defined as when the patient desires to participate in care but is unable to do so because of factors outside of his/her control. These could involve inadequate means of transportation, difficulties with schedule flexibility, etc. These patients are demonstrating nonadherence by current standards but would participate if circumstances allowed - outside pressures are influencing the decision to be nonadherent. Contrast that with voluntary nonadherence, defined as when the patient gives informed refusal to participate, and the patient is fully able to participate but actively chooses not to. This additional qualifying language could ensure protection for patients from being labeled and potentially dismissed due to circumstances outside of their control.
\end{abstract}

\section{INTRODUCTION}

As hospitals work toward shorter lengths of stay and shift to more outpatient care, patient care is being delivered in multiple settings. Each aspect of patient care can be important to the success of patient recovery and improvement including continual care settings like outpatient rehabilitation. Outpatient settings, however, present ethical challenges regarding whether health professionals should compel patients to stay engaged in care beyond the acute setting if the patient does not want this care. An additional consideration is what should be done if the patient does not participate. Clinicians may view such patients as noncompliant or nonadherent if they do not fully participate in their care. These labels, though, may not be fair to the patient, for there may be extenuating circumstances that prevent the patient from fully participating in care. Additionally, receiving a label of noncompliant or nonadherent can have long-term negative effects on a patient's ability to access care. The current standard of labeling a patient as nonadherent does not consider all aspects of patient decision-making; therefore new terms of voluntary nonadherence and involuntary nonadherence should be established. Having additional qualifying language and perspective when discussing these patients' inability to participate would be beneficial to the patient and society.

*Address correspondence to: Ryan Pferdehirt, D.Bioethics. Director of Membership and Ethics Education - Center for Practical Bioethics. Instructor of Bioethics - Kansas City University. 1111 Main Suite 500. Kansas City, MO 64105. Office: 816.979.1350. Cell: 412.370.4629. E-mail: rpferdehirt@practicalbioethics.org

+To cite this article: Ryan Pferdehirt . "Voluntary and Involuntary Nonadherence: Terminology for Labeling Patient Participation". The Journal of Healthcare Ethics \& Administration Vol. 7, no. 3 (Summer 2021): 1-7, https://doi.org/10.22461/jhea.1.71629

This work is brought to you for free and open access by the Institute of Clinical Bioethics (ICB) at Saint Joseph's University, Philadelphia, PA, U.S.A. It has been accepted for inclusion in The Journal of Healthcare Ethics \& Administration by the editorial board and an authorized administrator of the JHEA. For more information, please contact support@jheaonline.org 
Voluntary nonadherence occurs when the patient exercises informed refusal and knowingly chooses not to participate in care. Involuntary nonadherence occurs when a patient genuinely desires to fully participate but is unable to do so due to unforeseen circumstances, pre-existing limitations, or additional difficulties. Introducing the concepts of voluntary nonadherence and involuntary nonadherence with the patient would be helpful in trying to best meet the needs of patients. Health professionals need to recognize the difficulties many patients face when care is moved outside the acute setting. Focusing on the goal of upholding patient preferences and quality of life, as defined by the patient, allows patients to refuse outpatient physical therapy and rehabilitation as an ethical option. But the difference between voluntary and involuntary nonadherence can impact the patient/physician relationship, with voluntary nonadherence potentially causing an erosion of trust, so much so that the continuation of the relationship may not be in the best interest of the patient. The establishment of trust between physician and patient benefits both parties, as it enables patients to accept recommendations, improves outcomes, and decreases the likelihood the physician practices defensive medicine. ${ }^{12}$

\section{BENEFITS OF OUTPATIENT CARE}

Rehabilitation, specifically outpatient rehabilitation following a surgery, has been demonstrated to be a valuable aspect of continuation of care for several conditions. ${ }^{3}{ }^{4}$ Rehabilitation is important to the success of patient care. Studying comprehensive cardiac rehabilitation, Yohannes et al. found that, "twice-weekly cardiac rehabilitation over six weeks, on average, led to clinically significant improvement in quality of life, physical activity status, anxiety and depression in patients." ${ }^{5}$ Other rehab programs provide similar benefits, particularly for patients with critical illness. ${ }^{6} 7$ Although rehabilitation and physical therapy have demonstrated benefits, and declining such treatment could be deemed "unreasonable" by the clinical team, this decision, if made by a patient with capacity, should still be respected. Many factors go into declining medical treatments including social, personal, and financial considerations. The patient may not be acting unreasonably in declining therapy, but rather has made a decision that the medical team agrees is "reasonable" for that patient based upon the patient's particular circumstances. Understanding the decision making of patients is essential to understanding their reasons for accepting or declining treatment. As Atanelov et al. state, "The role and ultimate obligation of the physiatrist...is to thoroughly know and understand the patient as a person - including his or her interactions with family, employment, community, and environment." ${ }^{8}$ Understanding the motivations of a patient helps the provider to be consistent with the patient's goals of care. Patient engagement also helps in attaining patient goals of care. Lund, Tamm, \& Branholm found that patients who only occasionally participated in therapy felt, "...the professionals only occasionally asked for their participation...[and] the professionals gave them too little time and that they had little or no influence on the planning including the decision-making process." ${ }^{9}$ By engaging patients and supporting them in their decisions, providers can move away from a paternalistic approach to a model for shared decision-making.

Another consideration related to the patient's right to decline treatment is quality of life. Jonsen et al. describe quality of life as, "...that degree of satisfaction that people experience and value about their lives as a whole, and in its particular aspects, such as physical and psychological health." ${ }^{10}$ Patients, not medical professionals, determine acceptable quality of life. While the medical team may have their own opinions and biases, the role of the medical team is not to determine quality of life for the patient, as quality of life is a qualitative term that is unique to each individual patient. ${ }^{11}$ If the medical team determines quality of life for each individual patient, then respect for autonomy would diminish. Effective treatment plans occur when the medical team's

\footnotetext{
${ }^{1}$ Rosser, Walter W., Jan Kasperski. The benefits of a trusting physician-patient relationship.

${ }^{2}$ Hendren, Elizabeth M., and Arno K. Kumagai. A matter of trust.

${ }^{3}$ Yohannes, Abebaw et al. The long-term benefits of cardiac rehabilitation on depression, anxiety, physical activity and quality of life.

${ }^{4}$ Fan, Eddy. Critical illness neuromyopathy and the role of physical therapy and rehabilitation in critically ill patients.

${ }^{5}$ Yohannes, Abebaw et al. The long-term benefits of cardiac rehabilitation on depression, anxiety, physical activity and quality of life. Pg. 2810

${ }^{6}$ Fan, Eddy. Critical illness neuromyopathy and the role of physical therapy and rehabilitation in critically ill patients.

${ }^{7}$ Koo, K. K. et al. Prioritizing rehabilitation strategies in the care of the critically ill.

${ }^{8}$ Atanelov, Levan, et al. History of physical medicine and rehabilitation and its ethical dimensions. p 571

${ }^{9}$ Lund, Maria Larsson, et al. Patients' perceptions of their participation in rehabilitation planning and professionals' view of their strategies to encourage it. p. 158

${ }^{10}$ Jonsen, Albert, et al. Clinical ethics: a practical approach to ethical decisions in clinical medicine. p. 111

11 Jonsen, Albert, et al. Clinical ethics: a practical approach to ethical decisions in clinical medicine. p. 120
} 
recommendations and the patient's goals of care are upheld, but as previously stated, when this does not occur, the patient's preferences should be respected. The intention of recommending rehabilitation or physical therapy is improving quality of life for the patient, but this is improving quality of life as determined by the medical team. Ethical patient-centered care should rely on understanding the patient as a person to achieve goals of care and quality of life as defined by the patient, not as defined by the medical team.

Rehabilitation and physical therapy have clear benefits, so one might argue that the principles of beneficence and nonmaleficence are violated by allowing a patient to decline a treatment recommendation that would promote good for the patient and help eliminate potential future harms. But if the patient is informed, voluntary, and autonomous, the medical team would have to use hard paternalism, ${ }^{12}$ which is understood to be used when the physician believes that the patient is mistaken, confused, or irrational, therefore making it permissible to interfere in what the patient is requesting. ${ }^{13}$ Hard paternalism is typically viewed to be more ethically objectionable ${ }^{14}$ and therefore requires additional requirements to protect patient autonomy involving additional members of the medical or ethics consult team. Hard paternalism is only justified when certain conditions are met, including when, "The least autonomy-restrictive alternative that will secure the benefit is adopted." ${ }^{15}$ Regarding rehabilitation or physical therapy, effective and less autonomy-restrictive alternatives exist, including telehealth and home-based rehabilitation. ${ }^{16}$ Under these considerations, there is not enough support to override the patient's right to autonomy for not participating in rehabilitation and paternalism should be considered for, "actions that prevent major harms or provide major benefits while only trivially disrespecting autonomy." 17

When a patient refuses additional therapy including rehabilitation and physical therapy, the decision is likely not done with the intention of causing additional harm to oneself but rather the result of social and personal decision-making. Sometimes patients become overwhelmed, exhausted, and depressed by the prospect of continuous therapy and decline further treatment; the patient is not demonstrating true nonadherence but rather prioritizing additional aspects of quality of life. The medical team should work with the patient to understand the patient's goals of care more thoroughly, encourage engagement in continual therapy, and ultimately respect patient autonomy.

When the patient is not actively participating in the treatment plan, the patient can be determined to be nonadherent, but the medical team may likely agree the decision is justified. As the term nonadherence reflects a certain bias or judgment, the creation of additional qualifying terms to distinguish between these types of nonadherence is recommended: voluntary versus involuntary nonadherence. I will use the term involuntary, rather than nonvoluntary, to enforce distinction between action made with regret and those made without regret. ${ }^{18}$

\section{INVOLUNTARY NONADHERENCE}

The World Health Organization (WHO) defines adherence as "the extent to which a person's behavior - taking medication, following a diet, and/or executing lifestyle changes, corresponds with the agreed recommendations of a health care provider." ${ }^{19}$ Therefore, the failure to meet this definition could be defined as nonadherence. ${ }^{20}$ This is not a complete understanding of nonadherence, for nonadherence should involve the conscious and informed act of an individual to not participate in the recommendations. If a patient actively desires to fully participate in the recommendations but is not able to do so, he or she should not be determined to be nonadherent, but rather should be described as involuntary nonadherent. The term involuntary is necessary to convey the understanding that the patient is nonadherent but not by his or her own choice. The choice not to follow the recommended course of action is a decision that is being forced upon him or her, not made by freely. This concept has been explored by Clark, Ka rrington, \& Chilcot when they described intentional and nonintentional noncompliance stating, "intentional noncompliance refers to a premeditated effort to go against or not adhere to medical advice. Nonintentional adherence refers to unwillingly (i.e. forgetting)

\footnotetext{
${ }^{12}$ Beauchamp, Tom L., and James F. Childress. Principles of biomedical ethics. p. 217

${ }^{13}$ Dworkin, Gerald. Paternalism.

${ }^{14}$ Groll, Daniel. Paternalism, disagreements, and the moral difference.

${ }^{15}$ Beauchamp, Tom L., and James F. Childress. Principles of biomedical ethics. p. 222

${ }^{16}$ Clark, Sarah et al. Nonadherence in dialysis patients: prevalence, measurement, outcome, and psychological determinants. p. 35

${ }^{17}$ Beauchamp, Tom L., and James F. Childress. Principles of biomedical ethics. p. 221

${ }^{18}$ Barnes, Jonathan. The complete works of Aristotle.

${ }^{19}$ Sabaté, Eduardo et al. Adherence to long-term therapies: evidence for action. p. 3

${ }^{20}$ Cleemput, Irina, and Katrien Kesteloot. Economic implications of non-compliance in health care. p. 2129
} 
going against the advice given by a healthcare professional." ${ }^{21}$ While similar, prioritizing whether or not the nonadherence was intentional keeps the responsibility solely on the patient, rather than accounting for factors outside the patient's control such as a patient who lacks means of transportation to outpatient appointments. A patient who does not comply with a treatment plan because services are only offered during standard business hours and the patient is unable to take off work, or the patient lacks the required copays each week, or there are no available providers on the bus route, is technically "intentionally" being nonadherent they are intentionally prioritizing other factors over the treatment plan, such as staying employed or saving money for other bills. These types of external factors are different than forgetting to attend treatment, because the patient is aware that he or she is not participating in treatment, but feels unable to do so because of factors outside of his or her control. The use of the term involuntary shifts the cause for the nonadherence away from the patient and onto the additional factor(s) that contributed to the nonadherence.

\section{WHO GETS LABELED}

Patients of lower socioeconomic status are far more likely to not own a car, have inadequate means of transportation, not have schedule flexibility to make appointments, and have restrictions due to domestic responsibilities such as child or elder care. This may result in those patients more often being labeled as nonadherent.

When researching medication adherence specifically, Bazargan et al. found that medication adherence, while remaining an important issue for all older adults, was a particularly striking issue for underserved African Americans. ${ }^{22}$ Several variables including health literacy offer some explanation. ${ }^{23}$ Medication nonadherence was found to be common, with thirty-five percent stating they skipped taking medication on purpose but only eight percent stating they "forgot". ${ }^{24}$ This is even more striking when relating to lifestyle choice, with sixty-nine percent not adhering to specific food/drink instruction and seventy-three percent to activity instruction. ${ }^{25}$ Several reasons that are not voluntary may explain why a patient may be nonadherent to medical recommendations, including failure of communication, lack of comprehension, cultural issues, psychological issues, and psychosocial stress. ${ }^{26}$ All of these factors can easily be seen to disproportionately effect those of lower socioeconomic status. ${ }^{2728} 29$

The label of "nonadherent" also brings unhealthy stigma, which can then be unfairly attributed to an individual. This label gives the impression of a patient understanding the recommendation and actively deciding against it (voluntary nonadherence), which can cause providers to think, "No use putting taxpayers' or insurers' money into a "lost cause". Thus, terms "noncompliant" and "nonadherent" have developed negative nuances and connotations." ${ }^{30}$ (Narayan, 2016, p. 47) This mental labeling can then cause patients to feel "othered" and unwelcome, which will then impact their willingness to participate with recommendations moving forward, which would then reinforce the nonadherent label.

\section{DISMISSING A PATIENT}

One of the primary responsibilities of the medical team is to uphold patients' goals of care. Patients are autonomous beings and have the right to self-determination. Conflict arises when the medical team and patient are not in agreement regarding treatment and future treatments. Jonsen et al. state that, "Respect for the autonomy of the patient implies that a physician should, after explaining his or her own preferences, honor the patient's preference among medically reasonable options." ${ }^{31}$ The physician should attempt to fully explain the risks and benefits of a particular treatment so that the patient is able to properly express his or her

\footnotetext{
${ }^{21}$ Clark, Sarah et al. Nonadherence in dialysis patients: prevalence, measurement, outcome, and psychological determinants. p. 42

${ }_{22}$ Bazargan, Mohsen, et al. Non-adherence to medication regimens among older African-American adults.

${ }^{23}$ Brown, Marie T., and Jennifer K. Bussell. "Medication adherence: WHO cares?."

${ }^{24}$ Bazargan, Mohsen, et al. Non-adherence to medication regimens among older African-American adults. p. 5

${ }^{25}$ Bazargan, Mohsen, et al. Non-adherence to medication regimens among older African-American adults. p.5

${ }^{26}$ Kleinsinger, Fred. "Understanding noncompliant behavior: definitions and causes."

${ }^{27}$ Hodeib, Melissa, et al. Socioeconomic status as a predictor of adherence to treatment guidelines for early-stage ovarian cancer.

${ }^{28}$ Mehta, S., et al. Potential factors affecting adherence with HIV therapy.

${ }^{29}$ Manne, Sharon L. et al. Treatment adherence difficulties among children with cancer: The role of parenting style.

${ }^{30}$ Narayan, Mary. Noncompliant and nonadherent: time to retire these labels. p. 47

31 Jonsen, Albert, et al. Clinical ethics: a practical approach to ethical decisions in clinical medicine. p. 52-53
} 


\section{THE JOURNAL OF HEALTHCARE ETHICS \& ADMINISTRATION}

Vol. 7 | No. 3 (Summer 2021)

autonomy and give informed consent. Even when patients choose paths the physician disagrees with, the right to autonomy remains, as, "Some people may even choose medically 'unreasonable' options, such as declining life-saving treatments; after the physician attempts to persuade the patient to accept the recommended course, the patient's choice should be respected." ${ }^{32}$

Several reasons support firing a patient. These include inappropriate behavior, falsifying medical history, failure to pay bills, and verbal and/or physical violence. One of the most frequent reasons, though, is the patient's failure to comply with a treatment plan. ${ }^{33}$ Failure to comply is one of the more challenging reasons for dismissal because of the nature of patient nonadherence. True patient nonadherence is a reasonable cause for dismissal because if a patient actively chooses to not adhere to a treatment plan, then the physician may not be able to properly engage with that patient in the future. If trust does not exist between the patient and the physician, proper care might be impeded. The establishment of trust is essential for a proper relationship, so much so that the American Medical Association addresses it in the beginning of its Code of Ethics, in Opinion 1.1.1. It states,

The practice of medicine, and its embodiment in the clinical encounter between a patient and a physician, is fundamentally a moral activity that arises from the imperative to care for patients and to alleviate suffering. The relationship between a patient and a physician is based on trust, which gives rise to physicians' ethical responsibility to place patients' welfare above the physician's own self-interest or obligations to others, to use sound medical judgment on patients' behalf, and to advocate for their patients' welfare. ${ }^{34}$

The type of nonadherence addressed is specific: the patient has capacity, understands the situation, has resources and ability to follow the treatment, and consciously chooses to not comply. This is referred to as Informed Refusal. ${ }^{35}$ If the patient does not understand the treatment plan enough to properly follow, or socioeconomic factors impact the patient's ability to adhere, that is not informed refusal, but rather a subjective nonadherence. Many reasons for not adhering to treatment plan may exist, including lack of means, being overwhelmed by, or have competing goals. In fact, even physicians fail to complete courses of antibiotics and other treatment plans when prescribed to them. ${ }^{36}$ Therefore, failure to fully comply with physician recommendations should not be grounds for patient dismissal but rather potentially indicative of a patient that requires additional support.

\section{CONCLUSION}

A history of nonadherence does not necessarily mean that a patient will be nonadherent in the future. Rather, a patient's history can set an expectation or perceived likelihood of particular actions to occur but does not mean those actions necessarily will occur. The most important ramification of adding the qualifying language of voluntary and involuntary nonadherence is additional trust before labeling patients as nonadherent. This label can have negative effects for patients and negatively impact their ability and desire to access care going forward. Narayan puts this directly stating, "instead of labeling patients with these terms, clinicians should explore why their patients are not adhering to a plan that would promote their health and well-being" and how doing so can help identify "creative and evidence-based strategies that enable patient to be more engaged in developing workable care plans that promote their health." ${ }^{37}$ This additional terminology does not fix the situation of patients not being able to participate fully in care, but it promotes additional requirements to ensure that patients are not unfairly labeled nonadherent because of factors outside of their control. If a patient is deemed to be involuntary nonadherent, the logical next step would be to question what could be done to better support that patient and why he/she is unable to fully participate rather than stigmatize, label, and restrict his/her access to care. This new method of labeling patients involuntarily nonadherent can be an important additional protection for patients, potentially opening opportunities for the patient to receive access to additional resources or identify alternative treatment plans that enable adherence.

\footnotetext{
${ }^{32}$ Jonsen, Albert, et al. Clinical ethics: a practical approach to ethical decisions in clinical medicine. p. 53

${ }^{33}$ Sanfilippo, Joseph S., and Steven R. Smith. How do you dismiss a patient from your practice's care? p. 45

${ }^{34}$ American Medical Association. Code of Medical Ethics Current Opinions with Annotations.

${ }^{35}$ Ridley, Donald T. Informed consent, informed refusal, informed choice-What is it that makes a patient's medical treatment decisions informed.

${ }^{36}$ Capozzi, James, et al. Ethics in practice: Terminating the physician-patient relationship. p. 209

${ }^{37}$ Narayan, Mary. Noncompliant and nonadherent: time to retire these labels. p. 47
} 


\section{THE JOURNAL OF HEALTHCARE ETHICS \& ADMINISTRATION}

Vol. 7 | No. 3 (Summer 2021)

\section{BIBLIOGRAPHY}

American Medical Association. "Code of Medical Ethics Current Opinions with Annotations." (2016).

Atanelov, Levan, Steven A. Stiens, and Mark A. Young. "History of physical medicine and rehabilitation and its ethical dimensions." AMA journal of ethics 17, no. 6 (2015): 568-574.

Barnes, Jonathan. "The complete works of Aristotle. 2 vols. Vol. 1." (1984).

Beauchamp, Tom L., and James F. Childress. Principles of biomedical ethics. Oxford University Press, USA, 2001.

Bazargan, Mohsen, James Smith, Hamed Yazdanshenas, Masoud Movassaghi, David Martins, and Gail Orum. "Non-adherence to medication regimens among older African-American adults." BMC geriatrics 17 , no. 1 (2017): 1-12.

Brown, Marie T., and Jennifer K. Bussell. "Medication adherence: WHO cares?." In Mayo clinic proceedings, vol. 86, no. 4, pp. 304-314. Elsevier, 2011.

Capozzi, James D., Rosamond Rhodes, and George Gantsoudes. "Ethics in practice: Terminating the physicianpatient relationship." JBJS 90, no. 1 (2008): 208-210.

Clark, Robyn A., Aaron Conway, Vanessa Poulsen, Wendy Keech, Rosy Tirimacco, and Phillip Tideman. "Alternative models of cardiac rehabilitation: a systematic review." European journal of preventive cardiology 22, no. 1 (2015): 35-74.

Clark, Sarah, Ken Farrington, and Joseph Chilcot. "Nonadherence in dialysis patients: prevalence, measurement, outcome, and psychological determinants." In Seminars in dialysis, vol. 27, no. 1, pp. 42-49. 2014.

Cleemput, Irina, and Katrien Kesteloot. "Economic implications of non-compliance in health care." The Lancet 359, no. 9324 (2002): 2129-2130.

Dworkin, Gerald, "Paternalism", The Stanford Encyclopedia of Philosophy (Fall 2020 Edition), Edward N. Zalta (ed.), URL = <https://plato.stanford.edu/archives/fall2020/entries/paternalism/>.

Elwyn, Glyn, Dominick Frosch, Richard Thomson, Natalie Joseph-Williams, Amy Lloyd, Paul Kinnersley, Emma Cording et al. "Shared decision making: a model for clinical practice." Journal of general internal medicine 27, no. 10 (2012): 1361-1367.

Fan, Eddy. "Critical illness neuromyopathy and the role of physical therapy and rehabilitation in critically ill patients." Respiratory care 57, no. 6 (2012): 933-946.

Groll, Daniel. "Paternalism, disagreements, and the moral difference." American Philosophical Quarterly (2019): 57-70.

Jonsen, Albert R., Mark Siegler, and William J. Winslade. "Clinical ethics: a practical approach to ethical decisions in clinical medicine." (1982).

Hendren, Elizabeth M., and Arno K. Kumagai. "A matter of trust." Academic Medicine 94, no. 9 (2019): 12701272.

Hodeib, Melissa, Jenny Chang, Fong Liu, Argyrios Ziogas, Sarah Dilley, Leslie M. Randall, Hoda Anton-Culver, and Robert E. Bristow. "Socioeconomic status as a predictor of adherence to treatment guidelines for early-stage ovarian cancer." Gynecologic oncology 138, no. 1 (2015): 121-127.

Kleinsinger, Fred. "Understanding noncompliant behavior: definitions and causes." The Permanente Journal 7 , no. 4 (2003): 18. 


\section{THE JOURNAL OF HEALTHCARE ETHICS \& ADMINISTRATION}

Vol. 7 | No. 3 (Summer 2021)

Koo, K. K., Karen Choong, and E. Fan. "Prioritizing rehabilitation strategies in the care of the critically ill." Critical Care Rounds 8, no. 4 (2011): 1-7.

Lund, Maria Larsson, Maare Tamm, and Inga-Britt Bränholm. "Patients' perceptions of their participation in rehabilitation planning and professionals' view of their strategies to encourage it." Occupational Therapy International 8, no. 3 (2001): 151-167.

Manne, Sharon L., Paul B. Jacobsen, Kenneth Gorfinkle, Fern Gerstein, and William H. Redd. "Treatment adherence difficulties among children with cancer: The role of parenting style." Journal of Pediatric Psychology 18, no. 1 (1993): 47-62.

Mehta, S., Moore, R. D., \& Graham, N. M. (1997). Potential factors affecting adherence with HIV therapy. Aids, 11(14), 1665-1670.

Narayan, Mary. "Noncompliant and nonadherent: time to retire these labels." Home healthcare now 34, no. 1 (2016): 47.

Ridley, Donald T. "Informed consent, informed refusal, informed choice-What is it that makes a patient's medical treatment decisions informed." Med. \& L. 20 (2001): 205.

Rosser, Walter W., and Jan Kasperski. "The benefits of a trusting physician-patient relationship." Journal of Family Practice 50, no. 4 (2001): 329-329.

Sabaté, Eduardo, and Eduardo Sabaté, eds. Adherence to long-term therapies: evidence for action. World Health Organization, 2003.

Sanfilippo, Joseph S., and Steven R. Smith. "How do you dismiss a patient from your practice's care?" $O B G$ Management 27 no.6 (2015): 44-48.

Yohannes, Abebaw M., Patrick Doherty, Christine Bundy, and Ali Yalfani. "The long-term benefits of cardiac rehabilitation on depression, anxiety, physical activity and quality of life." Journal of clinical nursing 19, no. 1920 (2010): 2806-2813. 